\title{
La Commission des Recours des Réfugiés ou « l'intime conviction » face au recul du droit d'asile en France
}

Florence Greslier

\author{
(2) OpenEdition \\ Journals \\ Édition électronique \\ URL : https://journals.openedition.org/remi/4175 \\ DOI : $10.4000 /$ remi.4175 \\ ISSN : $1777-5418$ \\ Éditeur \\ Université de Poitiers \\ Édition imprimée \\ Date de publication : 1 octobre 2007 \\ Pagination : 107-133 \\ ISBN : 978-2-911627-46-6 \\ ISSN : 0765-0752 \\ Référence électronique \\ Florence Greslier, « La Commission des Recours des Réfugiés ou « l'intime conviction » face au recul \\ du droit d'asile en France », Revue européenne des migrations internationales [En ligne], vol. $23-n^{\circ} 2$ | \\ 2007, mis en ligne le 01 octobre 2010, consulté le 15 avril 2022. URL : http://journals.openedition.org/ \\ remi/4175; DOI : https://doi.org/10.4000/remi.4175
}

Ce document a été généré automatiquement le 15 avril 2022.

(C) Université de Poitiers 


\title{
La Commission des Recours des Réfugiés ou « l'intime conviction » face au recul du droit d'asile en France
}

\author{
Florence Greslier
}

1 L'Office français de protection de protection des réfugiés et apatrides (OFPRA) et la Commission des recours des réfugiés (CRR) ont été tous deux créés par la loi 52-893 du 25 juillet 1952, première grande loi en matière d'asile après la signature, en 1951, de la Convention de Genève sur les réfugiés. L'OFPRA est l'organe administratif de première instance, seul compétent pour instruire les demandes d'asile. La CRR est la juridiction administrative d'appel de l'OFPRA, que tout demandeur d'asile s'étant vu refuser le statut de réfugié en première instance est en droit de saisir.

2 Lors d'une enquête auprès de la Commission des recours des réfugiés ${ }^{1}$, nous nous sommes intéressée aux discours et aux pratiques des rapporteurs de cette juridiction administrative. L'objectif de la recherche était de repérer, entre déclarations politiques, législation et organisation institutionnelle, les logiques sous-jacentes au traitement de la demande d'asile. Il s'agissait de comprendre les différents enjeux qui déterminent l'attribution du statut de réfugié, en faisant l'hypothèse que les rapporteurs, de par leur statut, jouent un rôle central dans cette détermination. L'investigation s'est effectuée par divers moyens :

- observations d'audiences ;

- entretiens auprès de rapporteurs et de responsables de services de la CRR2.

- analyse de matériel non suscité : sites internet de l'OFPRA et de la CRR, « fiches pays » élaborées et rédigées par ces deux institutions, tracts et revendications écrites des employés de la Commission, etc. 


\section{À la commission : le rapporteur et son intime conviction}

3 Première en France en termes d'affaires jugées, cette juridiction procède à une étude complète de la demande du requérant, «comme s'il n'avait jamais été entendu ».En effet, "la Commission étant une "juridiction de plein contentieux", il lui revient d'estimer elle-même si l'intéressé peut bénéficier de l'asile. $»^{4}$

\section{Le rapporteur au centre du dispositif de recours}

4 La commission s'organise autour de 143 formations de jugement ${ }^{5}$. Selon la loi du 25 juillet 1952 (article 5), chacune d'entre elles est composée « d'un membre du Conseil d'État, président, désigné par le vice-président du Conseil d'État, d'un représentant du Haut Commissaire des Nations Unies pour les Réfugiés et d'un représentant du Conseil de l'OFPRA». Ces formations de jugements sont réparties en 10 divisions administratives au sein desquelles sont rattachés les rapporteurs. ${ }^{6}$

5 Le rapporteur est un cadre de catégorie A. "Toute l'instruction du dossier lui est confiée », rappelle le site internet de la CRR, qui définit ainsi ses missions : «Il assure toutes les phases de l'instruction des demandes [...], après refus du directeur général de l'OFPRA ; il rédige et présente un rapport de synthèse en séance publique de jugement ; il assiste aux délibérations et élabore le projet de décision ; il assiste certains présidents pour statuer par ordonnance sur les recours les plus manifestement infondés. » Et ses «qualités » attendues sont : « excellentes connaissances juridiques et bonnes niveau de culture générale » et "grande capacité d'analyse et de synthèse doublée de qualités rédactionnelles probantes $»^{7}$. Nous rapprocherons plus loin ces « qualités » des contrats de travail qui sont proposés aux rapporteurs.

6 L'action du rapporteur, officiellement désigné comme la « clef de voûte de la procédure d'appel » a donc mobilisé notre attention. Notre problématique s'inspire, d'une part, des travaux de Gérard Noiriel sur la place de la bureaucratie dans l'élaboration de critères d'identification en matière d'application du droit d'asile (Noiriel, 1991; 2001) et, de l'autre, de ceux d'Alexis Spire (2005) sur l'administration de l'immigration pendant les «trente glorieuses".

7 Ce dernier précise que sa démarche de recherche «repose sur l'hypothèse que la politique d'immigration se mesure aussi aux pratiques des agents qui la mettent en œuvre. En décidant du sort des étrangers qui se présentent aux guichets des préfectures, ceux-ci se livrent à un travail permanent de production, d'appropriation et de réinterprétation du droit. Ainsi, l'administration joue un rôle primordial comme instance de traduction du droit, autrement dit comme espace intermédiaire entre la loi et les étrangers » (Spire, 2005 : 11). À la différence des fonctionnaires de la Préfecture de Paris étudiés par cet auteur, les rapporteurs de la CRR ne travaillent pas en confrontation directe avec les demandeurs: l'instruction des dossiers se fait avant l'audience, sans rencontre avec les requérants. Toutefois, nous verrons qu'il y a pareillement pour ces agents le «travail permanent de production, d'appropriation et de réinterprétation du droit ", décrit par Alexis Spire, qui fait intervenir constamment leur subjectivité. 


\section{L'intime conviction des membres de la formation de jugement}

Au cours de l'enquête, il est apparu que la notion d'« intime conviction » fait partie de la terminologie propre à la Commission, invoquée tout autant par les rapporteurs que par les assesseurs (HCR et OFPRA). L'intime conviction est présentée comme inhérente à l'acte d'instruire ou de juger les demandes d'asile, et donc comme principe fondamental de cette juridiction.

- «Qu'est-ce qui fonde les conclusions de vos rapports d'instruction?

- Pour l'annulation ${ }^{8}$, c'est s'il n'y a pas de contradictions, si tout colle... Après c'est vraiment beaucoup d'instinct, de l'intime conviction, le truc c'est que nous on est dans l'écrit, on ne voit pas la personne.

- L'intime conviction?

- C'est le sentiment personnel, ma subjectivité... L'intime conviction du président par exemple, ça ne se discute pas " (Rapporteur)

- «L'intime conviction, c'est vraiment le fait d'être convaincu. On a le pouvoir de juger en son âme et conscience. Ça c'est un pouvoir qui n'appartient pas qu'au rapporteur. [...] À tous les niveaux, on est vraiment du côté de l'intime conviction, à moins d'avoir un dossier blindé. » (Rapporteur)

L'expression « intime conviction ", qui renvoie dans le langage courant à l'idée d'une certitude établie "au plus profond de ma conscience ${ }^{9}$, est en France une notion de justice pénale. Introduite par le législateur sous la Révolution française dans le premier code pénal de 1791 - la justice n'est alors plus rendue au nom du roi, elle désigne le transfert aux jurés de la charge de trouver une preuve dans leur for intérieur. Inscrite sous la forme d'un avertissement aux jurés d'assises dans le Code d'instruction criminelle de 1808, elle sera reprise dans le Code de procédure pénale de 1959: « La loi ne demande pas compte aux jurés des moyens par lesquels ils se sont convaincus; elle ne leur prescrit point de règles desquelles ils doivent faire particulièrement dépendre la plénitude et la suffisance d'une preuve ; elle leur prescrit de s'interroger eux-mêmes dans le silence et le recueillement, et de chercher dans la sincérité de leur conscience, quelle impression ont fait sur leur raison les preuves rapportées contre l'accusé, et les moyens de sa défense. La loi ne leur fait que cette seule question qui renferme toute la mesure de leurs devoirs: "Avez-vous une intime conviction?»(Article 353). Elle apparaît donc dans le droit pénal dans le serment des jurés d'assises, qui sont enjoints de " [se] décider d'après les charges et les moyens de défense, suivant [leur] conscience et [l'] intime conviction avec l'impartialité et la fermeté qui conviennent à un homme probe et libre [...], et de conserver le secret des délibérations, même après la cessation de [leurs] fonctions. » (Article 304). Dans les tribunaux de même, elle apparaît à l'article $427 \mathrm{du}$ même code comme "l'opinion profonde que le juge se forge en son âme et conscience et qui constitue [...] le critère et le fondement du pouvoir d'appréciation souveraine reconnu au juge du fait. » (Cornu, 1994 : 210).

Ainsi, en justice tant correctionnelle que criminelle, l'intime conviction fonde l'évaluation de la culpabilité d'un individu et le prononcé du jugement. Son usage au sein de la CRR, juridiction civile, et la présentation qui en est faite spontanément comme élément constitutif de l'action d'instruire et de juger les dossiers de réfugiés, personnes non supposées avoir commis une infraction, voilà qui nous surprend et nous interroge. L'emprunt au vocabulaire pénal ne participe-t-il pas au soupçon jeté ces dernières années sur le demandeur d'asile, coupable en puissance, suspect par sa sollicitation ? Ne renvoie-t-il pas, plus généralement, à la culpabilisation de la condition d'étranger? (Lochak, 1985). 
11 Le travail d'instruction des rapporteurs, comme nous le verrons plus loin, semble luimême s'inscrire dans une définition semblable à celle de l'activité d'un juge d'instruction. Il est présenté comme devant aider le rapporteur à se forger une intime conviction :

- « Moi je suis très particulier car je me veux juriste. À la Commission, une grande majorité se veut géopoliticien. Ils remontent pour voir s'il a menti, s'il manque des points, à quel niveau, ils vont reprendre les événements. Moi je vais dans la jurisprudence antérieure. Estce que ce cas a déjà été traité ? Et je me sers de ce cas pour me forger mon intime conviction.

- « Mon intime conviction»?

- C'est-à-dire, chaque cas est particulier, donc ce que je vais trouver va me servir de cadre intellectuel. Mais en même temps, je me dis que si un cas a déjà été jugé, à situation équivalente, le jugement ira dans le même sens. Donc si ça va dans le sens du requérant, je me dis que cette situation existe vraiment, donc je demanderai aussi l'annulation et je m'appuierai sur la jurisprudence. » (Rapporteur)

12 Dans l'expression «intime conviction» telle qu'elle est réappropriée par les rapporteurs de la CRR, tout se passe comme si le renfort de l'adjectif « intime » était destiné à produire un effet de légitimité incontestable de leur jugement. Ne serait-ce pas alors le signe d'une volonté d'objectiver une pratique qu'ils reconnaissent comme empreinte de subjectivité (et qui pour eux est parfois douloureuse), et ainsi la reconnaissance implicite qu'il n'y a finalement pas de critères objectifs applicables au traitement des demandes d'asile ? Jean-Michel Belorgey, président de section à la CRR, note quant à lui, dans un article consacré à l'intime conviction du juge, que ce dernier « dispose toujours, il est vrai, dans l'interprétation et la qualification des faits invoqués, d'une telle latitude que les solutions sont très largement aléatoires » (Belorgey, 2003 : 622).

Avant d'examiner cette question, nous formulerons deux hypothèses. En premier lieu, nous supposons que le contexte politique et discursif sur les étrangers en général est déterminant dans les pratiques quotidiennes des agents, en vertu du fait que «l'appropriation d'un principe juridique à un cas singulier n'est jamais une étape neutre » et qu'elle dépend non seulement « des règles d'interprétation établies par les hauts fonctionnaires, mais aussi des conditions dans lesquelles les agents intermédiaires sont amenés à adapter ces mêmes règles "(Spire, 2005 : 143). Or le constat d'une " poursuite de la spirale répressive ", dans une atmosphère croissante de suspicion envers des étrangers qui, toutes catégories confondues, sont réputés pratiquer l'abus et la fraude (Lochak, 1997 : 42-44), paraît aujourd'hui avoir une portée grandissante. Les agents publics chargés du traitement des demandes d'asile ne sauraient échapper aux effets d'un tel climat, depuis quelques années entretenu par la disqualification croissante des réfugiés dans les déclarations officielles (Morice et Rodier, 2005). En effet, la pénalisation discursive des réfugiés, d'emblée suspectés de vouloir profiter du système d'asile, appelle une injonction à distinguer les « bons » (ou « vrais ») des « mauvais » (ou «faux ») réfugiés. Un tel contexte pèse nécessairement sur les pratiques des agents de la CRR, qui reconnaissent aisément que, même en dehors de toute consigne formelle, il leur est difficile, voire impossible de passer outre $^{10}$. Cette injonction semble d'autant plus plausible que, d'un point de vue législatif, la catégorie de réfugié n'a pas bénéficié d'une définition précise.

La deuxième hypothèse porte sur l'illusion d'objectivité et de scientificité qui entoure le droit d'asile, du fait même de cette absence de définition. L'absence d'éléments juridiques objectifs qui permettraient le traitement des demandes d'asile entraîne, 
comme nous allons le voir, une tentative, par les acteurs, d'objectivation de leurs opinions. Jérôme Valluy explique ainsi l'usage de l'intime conviction en vue d'accorder ou refuser le statut de réfugié par l'absence d'éléments objectifs aussi bien dans la définition même du mot «réfugié » que dans celle du travail d'instruction: "Cette carence de fondements objectifs mais aussi les caractéristiques du droit dans ce domaine donnent à l'intime conviction du juge (comme à celle du fonctionnaire en premier examen), un rôle exceptionnel, et largement exorbitant du droit commun, dans la décision finale : loin d'être l'ultime arbitrage d'une instruction approfondie et d'un raisonnement juridique, tous deux étroitement dépendant du droit, l'intime conviction se substitue purement et simplement à l'une et à l'autre » (Valluy, $2004: 18$ ). Par-delà ce constat, qui était aussi auparavant celui de Jean-Michel Belorgey (2003) - et c'est cela qui sépare l'intime conviction de la justice rendue par le roi «au nom de Dieu »- les acteurs de la CRR, en se persuadant que les décisions préconisées se fondent sur des critères objectifs, ne tentent-ils pas de compenser l'inconfort moral de leur situation?

\section{Une impossible objectivation}

Par l'article premier de la Convention de Genève de 1951, est réfugiée toute personne étrangère qui, "craignant avec raison d'être persécutée du fait de sa race, de sa religion, de sa nationalité, de son appartenance à un certain groupe social ou de ses opinions politiques, se trouve hors du pays dont elle a la nationalité et qui ne peut, ou du fait de cette crainte, ne veut, se réclamer de la protection de ce pays ». Est laissée aux États signataires la liberté de mettre en place les modalités de leur choix dans l'identification des requérants éligibles au statut de réfugié. On voit ainsi que le sens qui sera donné à ce mot est à la fois d'une importance primordiale et d'une formalisation assez floue, notamment dans l'appréciation de ce que veut dire « craignant avec raison » et du sens donné au terme " persécution ».

16 Par la jurisprudence et les pratiques de traitement des dossiers de demande d'asile, il y a par conséquent tout un travail informel de définition de cette notion de réfugié, notamment par un processus de catégorisation, que nous pourrions définir comme un processus cognitif et idéologique consistant à prévoir le rangement des individus dans un ensemble de qualifications.

\section{Le poids de l'appartenance nationale et des stéréotypes associés}

17 Dans le cas français, la nationalité du demandeur est centrale dans le traitement de la demande d'asile. Or, dans le texte de la Convention de Genève, la nationalité n'est qu'un élément pouvant être source de persécutions parmi cinq. La place prise par la nationalité a été expliquée entre autres par Gérard Noiriel, qui énonce que le droit d'asile, son émergence et son application sont à penser dans une " nationalisation » des sociétés. Sous la III ${ }^{e}$ République, dès la fin du XIX ${ }^{e}$ siècle, la question des réfugiés va s'inscrire dans un enjeu qui devient progressivement central pour les sociétés modernes : celui du «national » et de l'« identité nationale». Il devient essentiel pour les États de définir les groupes de personnes pouvant bénéficier de leur protection. Cette définition passe par la nationalisation de la société : "Étant donné que c'est l'appartenance à l'État qui constitue le critère fondamental permettant de mettre en 
œuvre la politique de protection sociale, l'identification de la nationalité d'une personne devient un aspect décisif du travail juridico-bureaucratique de catégorisation " (Noiriel, 1991 : 277). Nous reviendrons sur cette idée à propos des fiches-pays produites par un service de la CRR, et qui nous furent présentées durant l'enquête comme centrales dans le travail d'instruction.

La notion très officielle de "pays sûrs" (pays dont les ressortissants sont, par définition, infondés à demander protection ailleurs) ne fait, elle aussi à son tour, que rendre plus déterminante la prise en considération de la nationalité dans l'évaluation de la demande d'asile, ou même plutôt du demandeur.

Quand ainsi le groupe national est pris pour entité pertinente, nous relevons dans les discours des rapporteurs un certain consensus quant aux traits distinctifs qu'ils attribuent à chaque groupe. Par exemple, si tous ne disent pas se sentir proches des demandeurs originaires de Mauritanie, tous nous indiquent les mêmes traits et la même présentation: les rapporteurs nous expliquent que les « Mauritaniens sont bien vus ici [à la Commission] » :

- « Pour la Mauritanie, alors là ils aiment bien : et là ce n'est pas la couleur, je crois que c'est

parce que ce sont des Noirs persécutés par des Arabes, ça, ça leur plait bien. » (Rapporteur)

L'exemple des demandeurs originaires de Chine revient de façon systématique dans les entretiens (cf. infra): tous les dossiers chinois seraient les mêmes et ce constat entraînerait des pratiques en matière d'instruction: il n'est plus nécessaire de les instruire, car ils sont d'emblée jugés «faux ». C'est d'ailleurs pour cette raison qu'on justifiera pour nous un jour l'absence de fiches-pays ${ }^{11}$ pour la Chine : « Ça ne vaut pas le coup.»

21 La nationalité du requérant apparaît alors comme une seconde nature, supposée immuable. Les rapporteurs parlent des demandeurs en les désignant par celle-ci, ajoutant ce qui fait leur spécificité à leurs yeux: "les Chinois sont menteurs", "les Moldaves sont touchants ", « les Angolais ne donnent que des faux [documents] ", etc.

L'assignation de traits distinctifs aux personnes passe ainsi beaucoup, dans le quotidien des instances d'évaluation, par leur appartenance d'origine à tel ou tel pays. L'objectivité d'un tel critère ne semble pas faire de doute pour les rapporteurs. Elle masque toutefois le caractère stéréotypique de la démarche, qui procède par généralisation à partir de fréquences apparemment incontestables de tel ou tel de ces traits. Cette réduction permet au rapporteur de pouvoir affirmer savoir distinguer les requêtes recevables des autres. Un tel processus de généralisation par réduction à des traits immuables n'est-il pas à situer dans un système politique et institutionnel spécifique duquel il semble difficile pour les rapporteurs de s'affranchir? Finalement, pourraient-ils faire autrement qu'avoir recours à cette réduction?

\section{Incursions permanentes de la subjectivité}

En vue de l'appréciation finale, il y a un certain consensus pour dire qu'un «bon » dossier serait un dossier " détaillé, bien ficelé », « clair, précis » :

- "Des événements détaillés qu'on ne peut pas inventer, quand c'est détaillé comme ça, là alors t'as envie de les aider. » (Rapporteure)

Il est aussi attendu des dossiers qu'ils soient « cohérents » :

- «Bien, disons par exemple que quand t'es recherchée par mandat d'arrêt, tu ne vas pas à

la police demander de l'aide. Il y en a qui disent ça, bon bien là c'est pas cohérent, même si 
t'es persécutée, mais que t'es recherchée, tu ne vas pas à la police, enfin il me semble. » (Rapporteur) comme autant d'éléments de preuve de la vraisemblance de sa demande, comme si $a$ contrario des dossiers trop concis, obscurs ou contradictoires devaient plaider en défaveur du requérant. Ils apparaissent et sont présentés comme allant de soi et bénéficiant d'un consensus à la Commission : à ce titre, ils n'ont pas à être justifiés et l'on ne sort pas de l'intime conviction. Les caractères d'un «bon » ou "mauvais » dossier ainsi définis peuvent alors être utilisés différemment selon la demande d'asile, selon que celle-ci est disqualifiée ou soutenue, et ce sans avoir à l'expliquer. JeanMichel Belorgey relève que du fait entre autres d'une certaine " propension au doute ", « si [tel dossier] est trop imprécis, il ne saurait naturellement convaincre. Mais s'il l'est (et il l'est rapidement) trop, cela ne peut naturellement qu'éveiller les doutes" (Belorgey, $2003: 620$ ).

is, outre ces éléments qui se voudraient objectifs, les rapporteurs admetten tous prendre en compte dans l'instruction des éléments tenant plus clairement de l'émotionnel. Ceux que nous avons rencontrés ont ainsi des catégories de dossiers pour lesquels ils se disent le plus sensibles. Ces catégories se définissent en général soit par la nature de la persécution (tous disent être particulièrement sensibles à la situation des femmes africaines invoquant les risques d'excision en cas de retour dans leur pays), soit par la nationalité du demandeur, à nouveau présente à ce niveau.

Ainsi, pour l'une ce sera les Mauritaniens : à l'évocation des requérants originaires de Mauritanie, son discours bascule dans un registre affectif, marqué par une identification importante au vécu de ceux-ci, « mes pauvres Mauritaniens».

- "Les pays de l'Est, j'ai du mal à comprendre. Après, j'aime beaucoup l'Afrique, notamment
la Mauritanie.
- Pourquoi?
- Je ne sais pas, certainement parce que les mouvements d'opposition n'étaient pas armés.
Mais après, je ne peux pas te dire pourquoi. Par exemple, je déteste le Sri Lanka, mais je ne
peux pas te l'expliquer. Il y a des pays dont tu te sens plus proche et d'autres pas. Moi je me
sens plus proche de l'Afrique sub-saharienne francophone et anglophone que des Sri Lankais
ou ceux du Pendjab. J'avais des copines qui adoraient bosser sur la Russie, la Tchétchénie,
moi ça me laissait complètement froide. Bon bien dans ce cas-là tu t'arranges avec ton chef
de section, genre "remets moi un peu d'africains". " (Rapporteure)

De telles préférences nous sont justifiées soit par l'intérêt personnel pour le pays (voyages privés, amis originaires de ce pays), soit par une identification à l'histoire, au vécu de ses habitants. Les rapporteurs disent alors comprendre les raisons qui les ont poussés à quitter leur pays : «J'aurais fait pareil moi à leur place.»

- "J'ai plus de facilités avec les dossiers d'Afrique, parce que j'adore l'Afrique, enfin je ne sais pas pourquoi. Après ça vient avec l'expérience aussi, il y a des pays que tu connais plus. Les dossiers des pays de l'ex-Union Soviétique sont passionnants parce que leur histoire est passionnante mais je n'y connais rien. Alors que l'Afrique j'y suis allée, je peux les imaginer dans leur contexte, dans leur climat. C'est essentiel pour comprendre de connaître leur contexte. » (Rapporteure)

La mise en avant de la compréhension de l'autre est en général introduite dans une analyse comparative entre les pays ou les demandeurs, comme l'illustre l'exemple qui vient d'être cité. Un rapporteur évoque l'existence de « modes » partagées par un grand nombre d'acteurs de la Commission : une nationalité va être pendant un temps l'objet d'une attention particulière, que ce soit en termes de rejet ou d'annulation.

Revue européenne des migrations internationales, vol. 23 - n² | 2007 

que dans celui de la distance ou de l'hostilité, du moins dans ce qui nous est dit: par exemple, on a envie de "faire passer un dossier ». L'entretien déjà évoqué nous indiquait que la rapporteure qui aime les dossiers africains cherche à éviter de traiter les autres. Nous verrons que cela s'accorde bien avec une certaine conception humanitaire du métier. Dans le cas contraire, une certaine distance vis-à-vis d'originaires de tel pays peut renvoyer à une supposée connaissance approfondie du pays, et se présenter alors ici comme une position objective fondée sur des éléments déterminants.

31 Mais, globalement, les rapporteurs perçoivent qu'une objectivation est impossible du fait même de ces incursions de l'affectif dans l'évaluation des dossiers de demande d'asile. Ils ont alors pour la plupart la sensation de faire du «bricolage», du " bidouillage », comme cela a pu nous être dit. Finalement, que ce soit par des éléments présentés comme objectifs ou par d'autres clairement subjectifs, il y a bien définition de critères d'évaluation, de catégories opératoires dans le travail d'instruction, qui dépasse alors la simple application du droit d'asile. Ce travail d'élaboration ne participe-t-il pas finalement à la définition de la notion de réfugié ? Cette définition, qui renvoie depuis quelques années à une dichotomisation "vrai »/ «faux » de plus en plus prégnante, et qui donne une place centrale à la nationalité du demandeur dans l'évaluation du risque de persécutions, se transmet par des moyens informels.

\section{La « culture maison »}

- «Certains [rapporteurs] peuvent être gênés, mais c'est plus par le poids de la culture maison sur telle ou telle annulation. Et puis si on ne demande pas trop d'annulations, c'est parce que la plupart des dossiers sont superficiels.

- Qu'est-ce que vous entendez par "culture maison"?

- On reçoit des informations, des notes qui nous disent que telle ou telle nationalité va très bien. On peut s'arrêter à ce discours public. Mais le rapporteur est indépendant, il peut aller faire d'autres recherches à côté. [...] Alors c'est fait avec des subtilités, des notes pour nous dire que tout va bien dans ces pays. Et puis paradoxalement, il y a des nationalités qui bénéficient d'un traitement plus large.

- C'est-à-dire?

- Les Sri Lankais, bon en France c'est le pays où ils bénéficient le plus du statut, et ça c'est connu, mais je ne peux pas l'expliquer. (Sourit)

- Connu par?

- Tout le monde, les Sri Lankais même. C'est ça que je voulais dire par culture maison, il y a une culture dominante qui influence les décisions, mais après on n'a pas de pressions sur les conclusions des rapporteurs. Donc il n'y a pas de pressions du tout, mais une culture dominante qui peut aller à l'encontre de la réalité. » (Rapporteur)

La « culture maison » de la CRR est composée des connaissances qui se transmettent, de façon informelle, des dirigeants aux rapporteurs et entre ces derniers. Celles-ci contribuent à une intelligibilité des pratiques de la Commission. La notion de «culture maison » se comprend alors comme l'ensemble de tout ce qui permet aux rapporteurs de définir leur place et leur mission à la CRR, par le moyen des pratiques et critères d'évaluation qu'elle véhicule. Elle se transmet par des notes de service (auxquelles nous n'avons pu avoir accès), par les relations quotidiennes entre collègues et par la formation qu'ils reçoivent.

Un élément de cette transmission a particulièrement retenu notre attention: un tutorat est mis en place à l'arrivée d'un nouveau rapporteur. Ce tutorat est assuré par 
une personne qui nous est présentée comme un "ancien». Ainsi, parmi les plus récemment embauchées (uniquement des femmes dans notre échantillon ${ }^{12}$ ), toutes nous parlent de leurs premiers temps à la Commission et de cette prise en charge par un « ancien », en précisant qu'il s'agit de quelqu'un « qui a plus d'expérience ».

En réalité, il est apparu que pouvait être considéré comme "ancien» un rapporteur salarié depuis un an seulement à la CRR. Cette bien faible ancienneté est à mettre en relation avec une certaine représentation dévalorisée du métier : "c'est pas difficile ", nous explique une rapporteure pour justifier le peu de temps passé en formation sur l'instruction même des dossiers. Un autre regrette l'absence de la reconnaissance d'un statut de rapporteur différencié de l'officier de protection de l'OFPRA. En outre, quel effet la féminisation du recrutement a eu sur la valeur supposée du travail ? ${ }^{13}$

La définition de l'ancienneté nous éclaire aussi sur la nature de ce qui se transmet: pour ces personnes, avoir de l'«expérience", ce serait être capable de reconnaître quand un requérant ment ou quand il s'agit d'un «faux réfugié ». Le tutorat nous est d'ailleurs présenté ainsi : il s'agit d'aider les nouveaux à repérer les « dossiers montés de toutes pièces » et ceux pour qui « ce n'est que du pipeau ».

Les composants de la «culture maison » ne se limitent pas à la suspicion vis-à-vis des requérants. À leur arrivée, toutes se souviennent que les premières phrases entendues ont été de trois ordres :

- par rapport aux requérants : « ce sont tous des menteurs »,

- sur le temps de travail : «tu verras tu n'auras plus de week-end »,

- sur leur propre avenir à la CRR : «barre-toi au plus vite».

Elles parlent alors d'une période de forte désillusion :

- «Et moi quand je suis arrivée, dans mon bureau, il y avait donc ma tutrice ${ }^{14}$ et un autre qui lui est là depuis 90, tu peux imaginer. Moi je suis arrivée avec tous mes idéaux et lui il disait que c'étaient tous des menteurs. Donc moi j'ai commencé comme ça, avec lui et donc quand je lui demandais son avis, il me disait à chaque fois que c'était du pipeau. Au début tu ne sais pas trop quoi regarder, sur quoi te baser. " (Rapporteure)

Nous n'avons pu, dans le cadre de l'enquête, connaître le cadre institutionnel de ce tutorat: était-il défini par l'institution? Si oui, combien de temps devait-il durer? Qu'était censé, officiellement, apporter le tuteur au rapporteur nouvellement recruté ? Ce qui ressort surtout des entretiens, c'est que le tutorat est laissé à l'entière responsabilité du tuteur et qu'il représente un vecteur de transmission de la « culture maison ", tant sur la teneur des dossiers que sur le rôle du rapporteur, ainsi réduit au repérage des dossiers à écarter, sur la base d'une présomption générale de mensonge.

\section{Les fiches-pays}

Pour leur travail d'évaluation, le Centre d'information géopolitique (CIG) met à la disposition des rapporteurs des fiches-pays - ou dossiers pays - définies ainsi sur le site internet de la Commission : « Les dossiers pays sont une présentation générale de la situation des principaux pays dont proviennent les demandeurs d'asile. Le CIG élabore ces documents en collaboration avec la délégation française du Haut Commissariat des Nations Unies pour les réfugiés (HCR). Un total de 41 dossiers pays sont ainsi élaborés soit par le CIG soit par le HCR (lien direct sur le site du HCR) et font l'objet de relectures croisées. La plupart de ces dossiers pays sont réactualisés chaque année. » 

chaque année. Outre le HCR, elles sont élaborées en collaboration avec l'OFPRA. La CRR a pris, il y a quelques années, le relais du HCR en rédigeant des fiches sur les pays non traités par cette institution. Ainsi, il apparaît que les rapporteurs ont à leur disposition des documents ayant bénéficié d'un regard partagé par des institutions censées apporter chacune un regard original sur la demande d'asile. Outre une bibliographie comportant un nombre important de sources communes, la présentation des fichespays est la même pour les trois institutions : elle suit, à peu de choses près, la même trame ${ }^{15}$.

De par sa présentation sur une base nationale, de par sa construction, de par ses choix, la fiche-pays implique une certaine approche de ce que sera un réfugié. La nationalité du requérant est essentielle : c'est elle qui sert de référence de base pour le choix de la fiche à consulter. Elle implique aussi une certaine conception de ce qu'est la persécution: cette dernière se définit non pas de manière générale mais de façon précise selon le pays, par la définition de groupes vulnérables et des agents de persécutions. Elle œuvre donc, par la relativité des cas, à rendre difficile le travail d'objectivation.

- «Les fiches-pays, c'est vraiment le reflet de la demande que l'on voit ici. Par exemple pour le Sri Lanka, on s'est surtout centrés sur les Tamouls. Je pense à la Mongolie par exemple, globalement il n'y a pas de soucis des droits de l'homme, mais par contre il y a quelques groupes vulnérables: je pense aux journalistes par exemple. Donc dans la fiche-pays, je vais y faire particulièrement attention, il va y avoir une entrée "média". Il n'y en aura pas forcément pour d'autres pays. Donc on oriente en fonction de la demande que l'on a. Au début, on a regardé les fiches du département d'État américain, qui sont vraiment bien, et on s'en est inspiré.

- Une fois que vous avez défini cela, est-ce que dans ces fiches vous vous positionnez sur ce à quoi il faut faire attention, sur les possibilités de refus et d'annulations?

- Disons qu'on va être très précis: pour la Mongolie par exemple, dans "groupes vulnérables", tous les journalistes ne le sont pas, donc je vais bien préciser quel type de journaliste est vulnérable. Voilà, donc en fait pour certains pays, on va être plus précis sur les groupes vulnérables et pour d'autres, ça va être autre chose. Et puis après, quand les rapporteurs viennent nous demander, on est amenés à dire, "bon ben non là, ce n'est pas vrai". » (Rapporteur du CIG)

Le Centre participe alors à la définition de la notion de "persécution ». Cette dernière est définie par les «agents de persécution » et les «motifs». Ainsi, une personne est persécutée à partir du moment où l'on a pu identifier par qui elle l'était. Cette façon de voir n'est pas sans influence sur les pratiques et le traitement des demandes d'asile. Par exemple, pour la situation en Haïti, il nous est expliqué que l'absence de connaissance des agents de persécution explique le faible taux d'attribution du statut de réfugié aux demandeurs d'asile de ce pays et le faible taux d'annulation des décisions de rejet en première instance. En même temps, les membres du CIG reconnaissent que l'insécurité y est généralisée et que tout le monde y est susceptible d'être persécuté.

- «Mais par rapport aux motifs de persécutions, par exemple, à la formation sur Haïti dont vous me parliez au début, on avait invité deux chercheurs, N. et aussi M., de la FIDH [Fédération internationale des droits de l'homme]. N. il disait en gros: "Si je me prends une balle dans la rue, ça ne veut pas dire que je suis persécuté par tel ou tel groupe, c'est peut-être tout simplement parce que j'étais là, à ce moment-là et c'est tout." Du coup on ne peut pas identifier les agents de persécution et par quoi moi, je suis persécutée. " (Rapporteure) 
43 présentation et par l'utilisation qu'elle invite à en faire, non seulement participe à la définition du travail de rapporteur (il s'agit de vérifier la véracité des faits présentés dans le dossier), mais est aussi une façon de demander à son tour au requérant de s'y conformer (pour ce faire, il doit pouvoir répondre aux items figurant dans les fiches). Dès lors, la vérification des connaissances des demandeurs sur leur propre pays entre dans le dispositif du rapport : lors de l'audience, pendant la lecture de ce dernier, il est fréquemment demandé aux personnes de présenter ou préciser ce qu'ils savent des orientations du pays, de l'histoire d'un parti politique par exemple.

Ensuite, par sa présentation détaillée des groupes vulnérables et des agents de persécution, la fiche définit des sous-groupes, dans la catégorie même des réfugiés. Elle remet ainsi en question l'approche individualiste du statut de réfugié telle qu'elle avait été conçue par la Convention de Genève. Le requérant doit alors répondre aux critères définissant, dans les fiches-pays, les groupes vulnérables, afin de pouvoir prétendre au statut de réfugié. Quelque peu paradoxalement, on lui demande d'assumer un traitement individualiste de son propre cas à partir d'une appartenance à telle ou telle catégorie prédéfinie par le CIG.

45 La question qui se pose enfin est de savoir comment il est possible de concilier les critères définissant un "bon dossier» (précision, personnalisation du récit) et la normalisation opérée par la Commission, quand elle définit des groupes auxquels le requérant doit appartenir pour être reconnu comme pouvant craindre " avec raison » des persécutions. C'est aussi entre autres dans cette contradiction que se situe la notion de « pays d'origine sûrs ».

L'incertitude de sa situation et du jugement qui sera porté sur celle-ci renvoie aux difficultés générales d'interprétation de l'article 1 de la Convention de 1951 et de la notion même de "persécution ». À quel moment notamment la persécution justifie-telle l'exil ? Quelles pièces convient-il alors de produire comme preuves de celle-ci ? Jean-Michel Belorgey remet ainsi en doute « la possibilité qu'un persécuté cherchant à quitter son pays en catastrophe pense d'abord, parvienne ensuite à se munir de semblables documents, ou de documents apparentés (photos compromettantes à raison de la prise ou de la détention desquelles on aurait été recherché ou poursuivi)» (Belorgey, $2003: 620$ ).

\section{Un travail sous contrainte}

47 La notion du temps se définit de façon singulière à la Commission. À travers le tutorat évoqué plus haut, ne transmet-on pas l'idée que l'expérience s'acquiert rapidement, que le travail d'instruction et les outils pour traiter les dossiers en distinguant les " vrais » des " faux " ${ }^{16}$ sont faciles à maîtriser, assez en tout cas pour être transmis à un autre après un an d'expérience? Cela nous amène à réfléchir sur le temps de la Commission et à prendre la mesure des contraintes sous lesquelles les rapporteurs ont à travailler.

Revue européenne des migrations internationales, vol. 23 - n² | 2007 


\section{"Faire du chiffre », l'exemple du déstockage de 2005}

48 À la fin de janvier 2005, les rapporteurs, interprètes et secrétaires, ainsi que des avocats, se mettent en grève pendant une semaine. Ils protestent contre l'injonction qui leur est faite de "déstocker ", c'est-à-dire de rattraper le retard pris dans le traitement des dossiers. Le stock à "liquider » est d'environ 50000 dossiers selon le gouvernement, 100000 selon les grévistes. Pour la direction, ce retard est dû à un nombre croissant de saisines de la CRR par des demandeurs d'asile déboutés ${ }^{17}$ et à une "procédurisation » croissante de la demande d'asile, qui s'expliquerait par la volonté des requérants de "gagner du temps » en faisant appel pour bénéficier du caractère suspensif du recours ${ }^{18}$.

La question posée à l'occasion de ce mouvement est celle des conditions de travail de rapporteurs sommés d'aller vite. Toutes les personnes rencontrées alors s'en plaignent, et ont l'impression qu'il leur est demandé de « faire du chiffre » :

- «On n'a aucun lien non plus avec les ambassades. La seule instruction (en riant) qu'on ait c'est "faites vite, avec le moins d'argent"! Ah oui et aussi "sans creuser". Ça, on n'est pas invités à creuser. Le stock, les flux, mais pas la qualité. » (Rapporteur)

Ils parlent de l'absence de moyens mis à leur disposition, face à un nombre trop important de dossiers à instruire par mois :

«Le plus minant c'est vraiment le manque d'infos, le temps, la pression que tu as pour instruire. » (Rapporteur)

51 Tous expliquent que la qualité du traitement des dossiers en pâtit en premier lieu, et ce constat rend pour la plupart d'entre eux d'un certain point de vue "insupportable » leur travail à la Commission :

- «Au quotidien c'est pesant, ce que ça heurte le plus en moi, c'est mon sens de la justice, je ne supportais plus, à la fin, de mal juger. » (Rapporteur)

La décision de déstockage justifiera alors la mise en place de pratiques institutionnelles officieuses et l'élaboration de catégories permettant un travail d'instruction plus rapide. Les rapporteurs évoquent entre autres la constitution des rôles ${ }^{19}$ comme moyen d'alléger le travail d'instruction :

- «Et puis il y en a des faciles comme ça... Les Chinois, les pauvres Chinois, souvent tous les dossiers étaient rédigés de la même façon, avec la même adresse de domiciliation, et puis de toute façon ils ne venaient même pas. Alors on les mettait à la fin des rôles pour alléger.

- Comment ça pour "alléger"?

- Pour que tu puisses mieux gérer les dossiers plus durs, plus conséquents, quand on constituait les rôles on mettait trois-quatre dossiers chinois pour équilibrer le rôle.

- En quoi les dossiers chinois permettent-ils d'équilibrer?

- Parce que tu n'as qu'une semaine de préparation pour quinze dossiers. Alors quand tu as quinze dossiers avec pour certains beaucoup de recherches, des pièces à mettre dans le dossier ou alors un problème de jurisprudence. [...] Et donc pour certains dossiers, tu as besoin de deux jours pour les faire et d'autres ça prend une heure, même une demie heure. Mais c'est fatigant en matière, c'est lourd. » (Rapporteur).

D'autres nous parleront de «petits » et de «gros» dossiers, les «gros » étant ceux à instruire, les « petits » ceux dont on peut prédire un rejet avant même l'instruction.

En dehors de tout jugement particulier sur l'organisation et de tout degré d'adhésion à ses objectifs, les rapporteurs repèrent ainsi pour nous les éléments de tension dans lesquels ils ont à exécuter leur travail. La thèse des autorités de tutelle, relayée par la direction est que les dossiers doivent être traités aussi rapidement que possible, afin de 
ne pas laisser aux demandeurs d'asile le temps de s'installer, pour invoquer ensuite leur bonne intégration au pays comme motif de régularisation en cas de rejet du recours. ${ }^{20}$

On peut alors se demander si l'accélération du rythme des examens de dossiers ne vient pas aussi servir une autre injonction indirecte, ne se nommant pas comme telle, qui serait finalement d'accepter le moins possible de ces dossiers. Les rapporteurs témoignent fréquemment que, par-delà leur "intime conviction", ils sont très conscients que ce serait mal vu, sinon impossible, de proposer plus d'annulations qu'ils le font ${ }^{21}$.

\section{Précarisation des contrats de travail}

De moins en moins de rapporteurs bénéficient du statut de fonctionnaire: actuellement, 65, $8 \%$ d'entre eux sont embauchés avec des contrats à durée déterminée (CDD) d'un $\mathrm{an}^{22}$. Cette proportion s'est accrue durant la période dite de "déstockage ", durant laquelle 90 rapporteurs ont été embauchés pour un an afin de renforcer les effectifs et traiter au plus vite les dossiers en attente.

Les contractuels évoquent une embauche faite à la hâte, sans qu'ils sachent sur quels critères se fondaient les recrutements.

"En septembre ou décembre 2003, je vois une annonce de recrutement à l'OFPRA dans Le

Monde. Je me souviens que l'annonce était drôle, je crois que je l'ai gardée, c'était écrit: "passionné pour les droits de l'homme..." En gros c'était "si vous y croyez..." Rétrospectivement je trouve ça bien drôle... Au début donc c'était un poste d'OP [officier de protection] à l'OFPRA. [...] Et puis j'ai eu un entretien avec le secrétaire général de la Commission. Ça a été rapide, c'était difficile de comprendre les critères de recrutement. Enfin à ce moment-là, c'était un petit recrutement: tous les âges, toutes les formations même si plutôt en droit et plutôt de l'international, et plutôt DEA/DESS, et des parcours différents. » (Rapporteure).

58 L'imprécision fait pendant à une formation qui ne semble pas suivre un cadre formalisé. Ainsi sur trois rapporteures recrutées à un an d'intervalle chacune, aucune n'a connu une même durée de formation. Les rapporteurs, recrutés pour un an lors du « déstockage » et maintenus à leur poste à l'issue de cette année (10 sur les 80 recrutés), ne peuvent pas non plus justifier les raisons du renouvellement de leur contrat de travail.

On peut alors s'interroger sur les raisons et les effets de ces imprécisions. Ajoutés à la précarité croissante des contrats de travail, les flous laissés tant dans l'embauche que dans la présentation des missions et dans la formation ne mettent-ils pas les rapporteurs dans une certaine insécurité quant à leur place et la définition de ce qu'il est attendu d'eux? Le manque de repères ne leur impose-t-il pas d'effectuer un travail de repérage et d'interprétation des attentes de la CRR dans ses non-dits, ses injonctions implicites?

60 Imprécision et précarisation paraissent finalement servir les dirigeants comme autant d'outils en matière de gestion de personnel et d'application de certaines orientations. Les rapporteurs en CDD supposent par exemple pour la plupart qu'accorder trop d'annulations entraîne une perte de "crédibilité » et le risque de ne pas voir son contrat renouvelé. Il est alors question de «rentrer dans le moule» de la Commission. Se pose alors la question de l'indépendance du rapporteur dans son travail d'instruction. En ce sens, la CRR se démarque des autres tribunaux administratifs mais 
aussi pénaux: quel autre juge voit le renouvellement de son contrat risqué d'être compromis par la nature du jugement rendu?

61 Toutefois, il ressort des entretiens que les discours oscillent en permanence entre d'un côté les doléances, la critique ou la condamnation des pratiques de la Commission - ici quant à la précarisation des contrats -, et de l'autre côté l'acceptation et la justification de pratiques au nom du travail. Ainsi, en même temps qu'ils expliquent ne pas pouvoir sans risque pour leur carrière statuer sur toutes les annulations qu'ils souhaiteraient, ils justifient le nombre important de rejets sur lesquels ils concluent par le fait que la plupart des dossiers sont faux :

Question sur l'existence de quotas en matière de conclusions :

- "Ah oui, ça c'est sûr. Je ne sais pas d'où, mais décider 5-6 annulations c'est énorme. Ce qui
est fou, c'est que rien n'est clairement dit, les présidents ils peuvent faire ce qu'ils veulent, si
les quinze dossiers du rôle méritent le statut, sont fondés, ils devraient tous pouvoir l'avoir.
Un jour j'ai eu neuf annulations, mais bon c'était au début. Quand j'en ai quatre ou cinq c'est
génial, aujourd'hui deux c'est bien. Quand tu n'as que des rejets, c'est déprimant, ça m'est
arrivé une fois, mais là j'ai vraiment cru que j'allais lâcher l'affaire. " (Rapporteure)

Précisons que cette remarque vient après l'énoncé, par la même personne, qu'à partir de son expérience, il faut reconnaître que la plupart des dossiers sont de "fausses demandes ", c'est-à-dire des demandes qui couvriraient une immigration économique.

\section{Faibles moyens et bricolages}

Auparavant, la CRR siégeait à Fontenay-sous-Bois. L'enquête que nous avons menée a eu lieu moins d'un an après son emménagement dans les nouveaux locaux de Montreuil. Les rapporteurs ont la sensation d'avoir été mal traités durant cette période : absence de connexion internet pour l'ensemble des rapporteurs, matériels informatiques défectueux, déménagements successifs de bureaux du fait des nombreuses embauches, etc. Tous disent avoir ressenti une absence de souci de leur direction quant à leurs conditions de travail, et ce fut vécu comme une dévalorisation de leurs place et rôle à la Commission.

Les conditions de travail et les sentiments qui les accompagnent sont des facteurs importants dans le traitement des demandes d'asile. Certains se plaignent d'avoir dû travailler chez eux, sur leur temps personnel, et à partir de leur propre connexion internet pour rechercher les éléments nécessaires à l'instruction - tandis que d'autres reconnaissent s'être limités aux informations les plus accessibles de la Commission. Tout cela ne peut que renforcer l'impression d'arbitraire et d'improvisation qui parfois s'attache au fonctionnement de l'institution.

Pour finir, le rapporteur est placé dans une situation de tension, où il a à mettre en cohérence des injonctions contradictoires entre objectifs institutionnels et pratiques au quotidien. Il est contraint de se mouvoir et d'osciller en permanence entre un encadrement vide (pas de formation initiale ni de trame d'instruction, précarité des contrats et donc insécurité de l'emploi) et des contraintes restrictives dans sa pratique au quotidien (nécessité de "faire du chiffre", caractère orienté des moyens mis à sa disposition pour instruire). D'où des remarques de ce type, pour relever les incohérences du système : «la Commission, c'est du n'importe quoi », «le droit d'asile c'est tout sauf du droit». Principalement chez les rapporteurs embauchés en CDD, s'exprime à un moment ou à un autre le souhait de ne pas rester à long terme à la CRR. 


\section{Le rapporteur comme sujet : entre souffrance et légitimation}

67 Pris dans un tel contexte politique et institutionnel, en proie à la difficulté de croire à l'objectivité de leur travail, déstabilisés par leur propre ambivalence, les rapporteurs vont à leur tour se voir contraints de bricoler avec leur propre subjectivité, entre sentiment de pouvoir et de dévalorisation, pour parfois, se réinventer comme sujets capables de procéder humainement à un traitement des demandes qu'ils qualifient volontiers par ailleurs d'inhumain.

\section{Le pouvoir : du « nous, on sait » à la tentation humanitaire}

Dans l'ensemble, les rapporteurs estiment que leur connaissance du dossier les met dans la meilleure position pour juger de la demande, pour "connaître la vérité » ("nous on sait si c'est vrai ou pas»), et que cette connaissance du dossier est directement liée au travail d'instruction : c'est par là qu'il est possible, dit l'un d'entre eux, de statuer « en toute connaissance de cause ».

Ils notent aussi que les présidents sont conscients de leurs compétences et, selon un autre, « $90 \%$ des décisions prises suivent les conclusions du rapporteur ». Ce sentiment est renforcé par une pratique institutionnelle qui donne une place centrale au rapport dans l'analyse du recours que fera la formation de jugement. En effet, seuls les présidents sont autorisés à lire le dossier avant les audiences. Les assesseurs, n'y ayant pas accès, reçoivent uniquement le rôle (liste des audiences de la demi-journée, qui indique entre autres informations les nom, prénoms, date de naissance, nationalité et numéro de dossier de la personne) quelque temps avant les audiences, et il leur appartient, s'ils le souhaitent, de faire des recherches uniquement sur les pays d'origine des requérants, et non sur leur demande d'asile spécifique. Cette différence entre les assesseurs et le président dans l'accès aux dossiers, qui n'est pas la seule ${ }^{23}$, renvoie à la place que prend le travail du rapporteur dans les décisions finales. Pour les assesseurs, le rapport de cet agent lu durant l'audience est le premier et principal accès à la demande du requérant. Précisons toutefois que l'autorisation faite aux présidents de consulter les dossiers avant les audiences n'est pas une obligation.

Le travail d'instruction ne connaît aucun contrôle au préalable de l'audience, et les rapporteurs ne reçoivent à aucun moment de leur formation et de leur carrière des instructions officielles sur la trame du rapport. Cette activité n'est soumise à aucune directive formelle. "Les actes d'instruction ne sont pas décrits par le droit, ni pour l'OFPRA, ni pour la CRR. Le témoignage de l'exilé ne fait l'objet d'aucun procès-verbal pouvant être relu et validé par l'exilé ou son conseil » (Valluy, 2004:21). L'absence de cadre légal donne alors une grande place au travail du rapporteur et à ses interprétations. Pour certains, c'est la garantie d'une certaine liberté.

71 Le contrôle vient après les audiences, lors de la rédaction des décisions. Il est exercé surtout par le Centre d'Information Juridique (CIJ), qui veille à ce que dans la formulation de la décision, il n'y ait pas de vices de formes, pouvant alors mener à un pourvoi en cassation. 
72 Le rapporteur n'a en outre aucune obligation dans les recherches qu'il mène pour l'instruction des dossiers.

- «Vous savez, la géopolitique c'est vraiment le sas du rapporteur, il peut y gagner ou y perdre du temps, de toute façon personne ne vérifie, il peut ne pas chercher, et du coup il finit plus tôt, et puis maintenant avec la "badgeuse" [les rapporteurs doivent pointer en début et fin de journée de travail], alors là c'est la porte ouverte! L'administration devrait forcer en disant: "Vous allez chercher sinon vous serez sanctionné." Vous savez quand dans une entreprise on encourage la paresse... [...] Mais il n'y a pas de sanction, et on ne leur dit pas que c'est prioritaire de mener des recherches géopolitiques, que c'est dans le profil de poste, ce n'est pas dans les directives gouvernementales, et puis bien sûr il n'y a pas d'outil pour que ça se fasse bien. [...] Les gens se rendent très vite compte que c'est sans risque s'ils ne font pas » (Rapporteur)

73 Conscients de cette marge de manœuvre, certains rapporteurs reconnaissent faire des omissions dans leurs rapports, ou insister sur un point plus qu'un autre pour qu'un demandeur obtienne une annulation. Aucun ne nous a présenté un cas inverse, à savoir où il aurait rédigé le rapport de telle sorte que celui-ci justifie clairement un rejet.

- «Vous savez l'instruction de fait est beaucoup plus dure que celle de droit, ici. Et les faits ça prend du temps, de les connaître, de les comprendre... Je veux dire, même si on veut faire de l'humanitaire, il faut avoir des infos.

- De "l'humanitaire"?

- En accordant le statut à une personne qui n'aurait pas dû l'avoir, mais parce que vous sentiez que même si le dossier était un peu vide, il y avait quelque chose derrière. Voilà vous bidouillez un rapport qui ne vaut rien sur le plan juridique, qui ne fera pas jurisprudence, qui ne dit rien sur le plan géopolitique, et puis vous concluez sur "en l'état du dossier, la personne peut craindre des persécutions en cas de..." Bon voilà c'est ça une décision humanitaire, on le fait tous [...]. Mais bon, on peut le faire mais en toute connaissance de cause, en connaissant la situation. Ce sont les situations où la personne a l'air au bout du rouleau, qu'on a l'impression qu'il faut faire quelque chose, qu'on est sa dernière chance, que si on ne le fait pas il va mourir. » (Rapporteure)

74 La conscience de leur pouvoir sur le jugement final vient du fait que les rapporteurs sont seuls à instruire le dossier. Ils revendiquent une reconnaissance plus importante de leur rôle et de leur place dans l'instruction et les audiences :

- «Il [le rapporteur] est autant juge que le président, il a vraiment la mainmise sur tout le dossier, lui il connait le dossier parfaitement. Il faut vraiment reconnaitre le rapporteur pour ce qu'il est. À savoir un juge, pas un simple administratif.

- Il faudrait qu'il ait une voix aux délibérés?

- Non, mais qu'il soit reconnu, qu'il ait la possibilité de discuter juridiquement avec l'avocat, en partie opposée.

- Comme un avocat général?

- Exactement, comme un juge d'instruction, qui viendrait dire le droit, les subtilités juridiques de l'affaire. Là on le voit simplement un représentant de l'OFPRA. Il faudrait qu'il soit vu comme pour éclairer les parties. » (Rapporteur)

75 Le juge d'instruction est, rappelons-le, soumis au principe d'intime conviction. Toutefois, la reconnaissance du pouvoir décisionnel des rapporteurs semble les placer à nouveau dans une situation de tension, voire de culpabilité : ce qui nous renvoie au poids de leur subjectivité.

\section{«Un simple pion ${ }^{24}$}

76 À d'autres moments, ces mêmes personnes font remarquer, contrairement à ce qui vient d'être dit, qu'ils ne sont finalement que de «simples fonctionnaires ", que les 
décisions se prennent ailleurs. Ainsi, dans leurs discours, on peut noter un va-et-vient entre d'un côté le fait que eux seuls « sauraient » et que les formations de jugement les suivraient à $90 \%$, et de l'autre que les "décisions ne leur appartiennent pas ", que "c'est aux formations de jugement de statuer». Aucun ne revendique une voix délibérative et certains avouent même qu'ils ne souhaiteraient pas faire partie d'une formation de jugement : «Je préfère ma place à la leur », conclut une rapporteure, en évoquant le temps du délibéré.

- «Moi je crois que je n'aimerais pas être membre d'une formation de jugement, c'est une grosse responsabilité, c'est quand même toi à la fin qui décides... Déjà que c'est vachement dur d'être rapporteur, tu en prends plein la gueule de tout le monde. Il y a même des avocats qui plaident contre toi.

- Quelle place a votre rapport dans les décisions prises?

- Je ne sais pas quel impact a mon rapport sur la décision, des fois je me dis aucun et puis en fait si je crois quand même un peu. C'est vachement dur de lire ton rapport et de conclure par une demande de rejet. T'es devant la personne et tu lui dis: "ce que vous me dites, c'est faux". Alors qu'il a raconté des trucs atroces, enfin bon c'est la demande d'asile de dire des choses atroces. Et c'est dur parce que tu n'es jamais sûre de ce que tu dis... C'est vrai que c'est dur. » (Rapporteure)

Les rapporteurs ne se pensent pas comme responsables des décisions. Par là, ils notent par exemple que ces décisions sont «injustes », que «c'est tout sauf du droit » mais, n'ayant pas à statuer aux délibérés, ils ne peuvent que constater cette injustice, n'en étant pas responsables.

Comment interpréter ce paradoxe entre sensation de pouvoir et sentiment d'une position de subalterne, qui n'aurait aucun moyen d'action? Plus généralement, que permet la parcellisation du travail à la Commission? Par "parcellisation», nous entendons le fait que les statuts et places de chacun des acteurs semblent cloisonner la responsabilité des uns et des autres dans la décision prise : le rapporteur instruit mais ne participe pas aux délibérés, les assesseurs jugent mais ne peuvent pas consulter les dossiers avant l'audience et ne signent pas la décision, le Président juge mais ne participe pas à l'instruction, il n'a pas l'obligation de lire les rapports avant les audiences et n'est pas rattaché directement à cette juridiction.

Christophe Dejours, par l'examen qu'il fait du processus de «banalisation du mal », qui serait l'adhésion d'individus à des pratiques jugées dans un autre contexte comme inhumaines, avance pour nous quelques éléments de réponses : « cette banalisation du mal n'est pas initiée par des motions psychologiques. Elle est initiée par la manipulation politique de la menace de précarisation et d'exclusion» (Dejours, 1998 : 171).

80 L'auteur poursuit: "La division du travail favorise incontestablement ce rétrécissement concentrique de la conscience, de la responsabilité et de l'implication morale. On ne maîtrise pas ce que les autres font, et l'on en dépend. On ignore même souvent ce qui se passe au-delà du monde proximal. [...] Pour d'autres travailleurs, au contraire, cet état de choses est utilisé comme un alibi, un abri, une défense contre l'angoisse de la conscience élargie, celle selon laquelle "homo sum, humani nihil a me alienim puto ${ }^{25 "}$ "(Dejours, 1998: 171-172). Cette banalisation mène alors à une « conscience morale rétrécie » (ibid.). 


\section{L'humanitaire : la marge de manœuvre du rapporteur}

81 Finalement, les rapporteurs rencontrés font le constat d'une situation professionnelle douloureuse. Cette "souffrance au travail», pour reprendre l'expression du même auteur, s'exprime, comme nous l'avons vu, à différents niveaux : dans la confrontation quotidienne aux récits de vie des requérants et aux événements qui motivent leur demande d'asile, dans le sentiment d'être instrumentalisé par une institution qui leur demande de plus en plus de faire du chiffre et sollicite de moins en moins leurs compétences professionnelles, dans le constat d'un traitement de la demande d'asile de plus en plus restrictif et subjectif. Dans les entretiens, ils évoquent cette souffrance notamment à travers une peur de s'endurcir, de se « blinder ».

Ainsi, ils disent s'être vite rendus compte, à leur embauche, du pouvoir qu'ils avaient sur la vie des demandeurs : ceux-ci viennent solliciter un statut « qui change leur vie » et les rapporteurs ont conscience des enjeux majeurs du recours, de son annulation ou de son rejet, sur l'avenir des demandeurs. Ainsi, qu'ils le veuillent ou non, ils participent à la prise de décision d'attribution du statut. Ils disent avoir du mal à supporter la dureté des situations auxquelles ils sont confrontés et des décisions qui sont prises. Ils disent craindre de ne plus être touchés par les histoires des requérants, et notent d'ailleurs, par la description d'audiences, les premiers signes de cette évolution.

- "Il y a vraiment des injustices sauf que là c'est la vie de la personne qui en dépend. Maintenant moi je vois bien, je commence à ne plus croire en grand-chose, je suis plus dure à convaincre. Je ne m'oppose quand même pas à des annulations sauf vraiment s'il y a des incohérences ou que tu as affaire un gros connard qui ment. Parce que ça aussi, c'est dur quand tu vois quelqu'un qui profite vraiment mais qui va passer. Je cherche vraiment à partir en fait parce que j'en peux plus, ça me fatigue. C'est dur, c'est difficile de faire ton travail, parce qu'en plus tu penses à la personne, mais en même temps ça te détruit. Moi cela m'est arrivé de finir en pleurs, même souvent, après les audiences, parce qu'une décision n'est pas passée, parce que des assesseurs ont été humiliants. Des fois c'est terrible, les questions qu'ils posent... [Silence] En fait c'est difficile de concilier l'humain avec l'administratif, le droit. » (Rapporteure)

Les rapporteurs ont le sentiment de se retrouver seuls face au traitement des demandes de recours et face à leur part de responsabilité qu'ils ne peuvent finalement que constater.

- «Le plus dur c'est quand tu rédiges les décisions ${ }^{26}$ : là tu te refais les séances, tu as vu la personne, tu as pu te faire une idée. Alors tu te retrouves avec tes doutes, les décisions prises, sur ta formulation dans le rapport. Tu te dis: "j'aurais pas dî dire le viol comme ça, parce qu'en fait peut-être c'était vrai". Mais en fait plus tu lis des récits, plus ça devient abstrait, théorique. Comme le viol, tu lis le récit et puis tu te dis: "bon voilà et puis après elle a été violée" " (Rapporteure)

84 Au regard de ce que nous avons présenté précédemment, ce sentiment de solitude n'est-il pas renforcé entre autres par le statut de ces rapporteurs, contractuels pour la plupart, qui conditionne en permanence leur lien à la juridiction au nom de laquelle ils sont censés instruire et conclure?

On peut alors dans ce contexte tenter de comprendre les pratiques, décrites par nombre d'entre eux, qui consistent à «faire de l'humanitaire ». Par là, n'y a-t-il pas une tentative de se réapproprier son rôle en le rendant positif, de rendre supportable ce qu'ils décrivent comme insupportable? 
comprend aussi peut-être mieux les incursions de l'affectif dans le traitement des dossiers, incursions repérables à travers l'usage d'un lexique de la possession : «J'ai obtenu... », "J'ai eu mon annulation », « On est content quand on arrive à faire passer ses dossiers ». Souvent, ils expliquent qu'il s'agit durant les audiences de "convaincre les juges", de "faire passer ses dossiers ». N'y a-t-il pas là une tentative d'une réappropriation de son métier comme sujet, pourtant voué à une mission difficile, sinon impossible dans le cadre où elle s'exerce actuellement?

\section{Conclusion}

À l'issue de ces réflexions, nous pouvons revenir en conclusion sur l'usage de l'expression "intime conviction", envisagée comme tentative de légitimation et d'objectivation des pratiques d'agents dont la fonction est de contribuer à la prise de décisions mettant en jeu l'existence de personnes. La légitimation passerait, comme on l'a suggéré, à la fois par une mise à distance de leur place dans l'institution - ils se positionnent en observateurs de ce qui se fait, eux "savent» mais personne ne le reconnaît - , mais aussi par la présentation de critères objectifs et quasiment scientifiques, garantissant leur validité juridique.

Dans un récent rapport, Anicet Le Pors préconise la création d'un «statut d'emploi ${ }^{27}$ de rapporteur à la CRR ». Cette proposition, qui permettrait selon l'auteur la constitution impérative d'un corps de fonctionnaires, permettrait de dépasser la lassitude exprimée par les rapporteurs : «La question principale qui se pose à leur propos est de savoir si, en dépit de l'intérêt de la fonction exercée (l'instruction, phase essentielle de la procédure de demande d'asile), celle-ci peut être organisée en carrière sur plusieurs décennies d'une même vie professionnelle. Il se dégage de tout ce qui précède que la réponse est négative. Cette opinion est d'ailleurs corroborée par le sentiment fréquemment exprimé par les rapporteur(e)s que ce travail, aussi intéressant soit-il, finit par entraîner une lassitude au bout d'un certain temps (5 à 10 ans) à laquelle la faiblesse des débouchés et les limites de la mobilité ne peuvent remédier. " (Le Pors, $2006: 62$ )

Nous pouvons alors nous demander si l'usage des termes "intime conviction» ne viendrait pas témoigner de la parodie de justice dans laquelle la Commission des Recours des Réfugiés semble être prise. La Commission, par le statut de ses agents, par son lien avec l'OFPRA et par d'autres éléments encore, se différencie clairement des autres juridictions administratives françaises. Parler d'intime conviction à la CRR ne renvoie ainsi à aucune garantie d'indépendance de l'instruction et du jugement. Bien au contraire, l'étude du travail des rapporteurs et des conditions de celui-ci semble surtout révéler le maintien, par les pratiques décrites, de la part d'arbitraire de l'État sur le traitement des demandes d'asile et plus généralement vient corroborer l'hypothèse d'un pouvoir toujours plus discrétionnaire de celui-ci sur l'appréciation de la demande d'asile dans un sens toujours plus restrictif. 


\section{BIBLIOGRAPHIE}

CORNU Gérard (1994) Vocabulaire juridique, Paris, PUF, 4 ème éd, 968 p.

BELORGEY Jean-Michel (2003) Le contentieux du droit d'asile et l'intime conviction du juge, La Revue administrative, $\mathrm{n}^{\circ}$ 336, pp. 619-622.

BOURDIEU Pierre (1979) La distinction : critique sociale du jugement, Paris, éditions de minuit, $670 \mathrm{p}$.

Commission des Recours des Réfugiés (2006) Bilan statistique de l'activité de la Commission pour le premier semestre 2006. Consultable sur :

http://www.commissionrefugies.fr/presentation 4/rapports activite 139/statistiques 140/ activite commission 2093.html

DEJOURS Christophe (1998) Souffrance en France, Paris, Le Seuil, 197 p.

ESGAULX Marie Hélène (des), DENIAUD Yves, BONREPAUX Augustin (2005) Mission d'évaluation et de contrôle, pour une prise en charge plus rigoureuse des demandeurs d'asile, Paris, Rapport d'information $n^{\circ} 2448$ : Commission des Finances, de l'économie générale et du plan, juillet 2005, 165 p.

FASSIN Didier, MORICE Alain et QUIMINAL Catherine (dir.) (1997) Les lois de l'inhospitalité, les politiques de l'immigration à l'épreuve des sans papiers, Paris, La Découverte/Essai, $288 \mathrm{p}$.

Collectif, GISTI (1999) Asile(s) degré zéro, Plein droit nº 44, décembre 1999.

GRESLIER Florence (2005) Le traitement de la demande d'asile à la Commission des Recours des Réfugiés : catégorisations des réfugiés et pratiques des rapporteurs, Mémoire de Master de Recherche, Université Denis Diderot - Paris 7, UFR des Sciences Sociales, Département Sociologie et anthropologie de la migration et des relations interethniques, $151 \mathrm{p}$.

KEVONIAN Dzovinar (2005) Enjeux de catégorisations et migrations internationales : Le Bureau International du Travail et les réfugiés (1925-1929), Revue Européenne des Migrations Internationales, 2005 (21) 3, pp. 95-124.

LE PORS Anicet (2006) Rapport sur la situation statutaire des personnels de la Commission des Recours des Réfugiés, 120 p. Consultable sur :

http://www.commission-refugies.fr/presentation 4/actualites 5/situation statutaire 2108.html LOCHAK Danièle (1985) Étrangers de quel droit ?, Paris, PUF, coll. Politiques d'aujourd'hui, 256 p. MORICE Alain (2004) Des dizaines de milliers de réfugiés tenus à distance. L'Europe enterre le droit d'asile, Le Monde Diplomatique, mars 2004.

MORICE Alain, RODIER Claire (2005) Classer-trier migrants et réfugiés : des distinctions qui font mal, Hommes et Libertés - Revue de la Ligue des Droits de l'Homme, $\mathrm{n}^{\circ}$ 129, janvier - mars 2005, pp. 58-61.

NOIRIEL Gérard (2001) État, nation et immigration. Vers une histoire du pouvoir, Paris, Belin, collection « socio-histoires », $399 \mathrm{p}$.

NOIRIEL Gérard (1991) La tyrannie du national : le droit d'asile en Europe, 1793-1993, Paris, CalmannLévy, $355 \mathrm{p}$.

RYGIEL Philippe. (dir) (2004) Le bon grain et l'ivraie. L'État-nation et les populations immigrées, fin XIX ${ }^{\text {- }}$ début $X^{e}$ siècle, Paris, Presses de l'École Normale Supérieure, 170 p. 
SPIRE Alexis (2005) Étrangers à la carte : l'administration de l'immigration en France (1945-1975), Paris, Grasset, $402 \mathrm{p}$.

SULLEROT Evelyne (1968) Histoire et sociologie du travail féminin, Paris, Gonthier, 397 p.

VALLUY Jérôme (2004) La fiction juridique de l'asile, Plein Droit, nº 63, Paris, GISTI, pp. 17-22.

\section{NOTES}

1. L'enquête a eu lieu entre septembre 2005 et avril 2006, dans le cadre du Master « Sociologie et anthropologie de la migration et des relations interethniques » de l'Université Paris 7 - Denis Diderot - cf. Greslier (2006).

2. En tout, treize entretiens ont été menés pour cette enquête: huit auprès de rapporteurs, quatre auprès de représentants du Haut Commissaire des Nations Unies pour les réfugiés (HCR) et un auprès d'un représentant du conseil de l'OFPRA.

3. Entretien janvier 2006, rapporteur du Centre d'information Juridique de la CRR.

4. Site CRR, « Le recours ».

5. Source : http://www.commission-refugies.fr/presentation 4/

6. En février 2005, le nombre en équivalent temps plein (etp) de rapporteurs en section était de 152,8; en février 2006 ce nombre passe à 68,6 (CRR, 2006).

7. Site internet de la CRR, www.commission-refugies.fr

8. Le terme "annulation " s'applique à la décision de rejet par l'OFPRA : il signifie donc l'octroi du statut de réfugié. Notons que la confirmation de la décision de rejet prise par l'OFPRA sera nommée « rejet » par la CRR, dans le sens de rejet du recours.

9. Le Petit Larousse, 1995.

10. Rappelons toutefois pour nuancer notre propos que l'injonction de distinguer les « vrais » des «faux » et la méfiance à l'égard des réfugiés n'est pas nouvelle : Dzovinar Kévonian remarque, à propos de l'accession des réfugiés au « passeport Nansen » que, déjà en 1926, « parmi les raisons qui expliquent le peu d'application réelle du certificat, domine la méfiance des autorités à l'égard des populations réfugiées et l'impossibilité où elles disent se trouver de "distinguer entre les demandes de réfugiés de bonne foi et celles de personnes qui, quoique ayant droit à un certificat, avaient réussi à obtenir la délivrance d'un certificat dans des buts irréguliers." [Citation : Mémorandum soumis à la conférence intergouvernementale, 10-11 mai 1926, ASDN, FMN, C1470] », (Kévonian, 2005 : 102).

11. Les fiches-pays sont les documents élaborés par le Centre d'information géopolitique de la CRR, résumant un certain nombre d'informations sur les pays d'origines des requérants et mis à la disposition des rapporteurs pour leur travail d'instruction.

12. Les récents recrutements à la CRR ont été marqués par une forte féminisation des embauches. Un rapporteur, travaillant depuis plus de 5 ans à la CRR et entré après concours sur titres, nous explique que l'augmentation de la présence féminine aurait entraîné une augmentation des annulations et un changement de la jurisprudence pour les demandes fondées sur les mariages forcés des femmes, sur les craintes d'excision pour les enfants en cas de retour dans le pays d'origine. Ce changement nous est alors présenté comme l'arrivée « d'une autre sensibilité ».

13. Dans La Distinction (1979), P. Bourdieu avance l'idée d'une dévaluation des professions supérieures ou d'élite dès lors que les femmes parviennent à y accéder. Ce processus de déclassement s'inscrit alors dans celui que connaissent déjà les titres scolaires, dévalorisés par l'appartenance sexuée des diplômées (cf. aussi Sullerot, 1968).

14. En poste à l'époque depuis un an.

15. Trame de la fiche-pays de la CRR : un historique et la fiche proprement dite : situation générale, économie, population, langues, éducation, religion, médias, structure politique, 
élections, partis politiques, syndicats, associations et groupes de défenses des droits de l'homme, justice, service militaire, forces de sécurité, agents de persécution, détention et torture, groupes vulnérables, réfugiés, documents d'identité et déplacements. Enfin, viennent une chronologie et une bibliographie.

16. Une rapporteure précise qu'il s'agit pour elle de «repérer le faux du vrai » : la nuance de cette formulation, «le faux du vrai » ne revient-elle pas à dire que dans tous les dossiers, même ceux qui bénéficient au final d'une annulation, il y a de toute façon une part de faux, qui apparaît comme quasi inhérente à toutes les demandes d'asile?

17. Au premier semestre 2006, $86,47 \%$ des rejets de l'OFPRA font l'objet d'un recours à la CRR ; pour l'année 2005 , ce chiffre était de $85,66 \%$. (CRR, 2006)

18. Rappelons que le recours devant la CRR cesse d'être suspensif dans le cas d'une procédure prioritaire.

19. Les rôles sont la liste des dossiers qui seront auditionnés durant une audience, soit sur une demie journée. Un rôle représente en général quinze recours.

20. L'attractivité supposée de la France pour les demandeurs d'asile serait ainsi due au temps laissé à l'installation durant la procédure. Ainsi dans un rapport de la Commission des finances, de l'économie générale et du plan, est-il noté : «Il est difficile de remettre en question la faculté de réexamen. Cependant, il est possible de la rendre moins attrayante pour ceux qui l'utilisent à des fins dilatoires pour demeurer sur le territoire national. » (Esgaulx (des), Deniaud, Bonrepaux, $2005: 17)$

21. Pour le 1er semestre 2006, sur l'ensemble des décisions rendues par la CRR, 15,7 \% étaient des annulations, soit dans le détails : 1,17\% d'annulations pour accord de protection subsidiaire et $14,56 \%$ pour annulation et accord du statut. (CRR, 2006)

22. Au 1er mai 2006, sur les 85 rapporteurs de la CRR, 29 étaient titulaires, 56 contractuels. (Le Pors, $2006: 27$ )

23. Par exemple, seul le président signe la décision finale, alors que tous ont participé aux délibérés.

24. Expression employée par un rapporteur lors d'une pause après une audience.

25. « Homme je suis, rien de ce qui est humain ne m'est étranger. »

26. Il incombe au rapporteur de rédiger l'ensemble des décisions prises par le jury durant les délibérés à l'issue d'une séance. Elles sont finalement signées par le président du jury.

27. «Le statut d'emploi est, en quelque sorte, un statut de module de corps. Il doit préciser le corps et le grade auxquels doivent appartenir les fonctionnaires susceptibles d'être nommés à ces postes. L'appartenance à un corps de fonctionnaires est donc une condition préalable, ce qui exclut l'affectation d'un agent non titulaire dans un statut d'emploi. » (Le Pors, 2006 : 65).

\section{RÉSUMÉS}

Sur la base d'une enquête menée auprès des rapporteurs de la Commission des recours des réfugiés (CRR), cet article propose de repérer les logiques sous-jacentes au traitement de la demande d'asile. L'intérêt est centré sur les mécanismes d'appropriation et d'interprétation du droit d'asile par ces agents, qui instruisent les demandes de recours et produisent pour l'audience un rapport à la fin duquel ils ont à conclure sur l'annulation ou le maintien de la décision de l'office français de protection des réfugiés et apatrides sur l'attribution du statut de réfugié. Nous nous attachons à expliquer le contexte politique et institutionnel qui contraint le 
travail des rapporteurs. L'analyse de l'organisation du travail à la CRR et des conditions d'exercice de celui-ci permet de révéler les tentatives par ces agents de légitimation et d'objectivation de leur pratique, leurs difficultés rencontrées dans l'accomplissement de leurs tâches et de soulever des questions plus générales que posent les procédures en matière d'application de droit d'asile.

The French Refugee Appeals Board or the "Inner Conviction" facing the Decline of Asylum Rights in France. Based on the results of a series of interviews with agents from the French Refugee Appeals Board, this article attempts to reveal the underlying logic of the asylum seeking process. The article focuses on the application and interpretation of the asylum laws as practised by these agents, who investigate an appeal and present to a hearing a report which concludes in the quashing or confirmation of the decision of the French Refugee and Stateless Protection office to attribute refugee status. We endeavour to explain the political and institutional context which determines the work of the agents. An analysis of the organisation of work at the French Refugee Appeals Board and the conditions of practice there permits to look at the agents' attempts to legitimise and objectify in their work-practice, their difficulties met in the fulfilment of their tasks and to generally raise questions concerning the asylum rights procedures in France.

La Comisión de Recursos de los Refugiados o la «íntima convicción» ante el retroceso del derecho de asilo en Francia. Sobre la base de una encuesta dirigida a agentes de la Comisión de Recursos de los Refugiados (CRR), este artículo propone identificar las lógicas subyacentes en el tratamiento de la petición de asilo. El estudio se centra en los mecanismos de apropiación e interpretación del derecho de asilo utilizados por dichos agentes. Se trata de explicar el contexto político e institucional que influye en el trabajo de los agentes encargados de instruir las peticiones de recurso. La instrucción de las peticiones incluye la elaboración de un informe que será presentado a la audiencia y donde se concluirá la anulación o la confirmación de la decisión de la Oficina francesa de protección de los refugiados y apátridas relativa a la atribución del estatuto de refugiado. El análisis de la organización del trabajo en el seno de la CRR así como de las condiciones de su ejercicio revela ciertas problemáticas: los intentos de los agentes por legitimar y racionalizar sus prácticas, las dificultades ante las que se encuentran los agentes en el cumplimiento de las tareas que les son encomendadas $\mathrm{y}$, finalmente, ciertas cuestiones más generales ligadas a los procedimientos de aplicación del derecho de asilo.

\section{AUTEUR}

\section{FLORENCE GRESLIER}

Doctorante à l'Unité de recherche Migrations et Société (UMR 7032), Université Paris 7 - Denis

Diderot Casier 7027 - 2 place Jussieu, F-75251 Paris Cedex 05. E-mail : fgreslier@yahoo.fr 